\title{
Droits de propriété et d'usage sur la terre. Une étude statistique des recours contentieux en matière de fermage
}

Property rights and using rights on farmland: a statistical study of litigation concerning farm tenancy

\section{Romain Melot}

\section{OpenEdition}

Journals

\section{Electronic version}

URL: http://journals.openedition.org/economierurale/4402

DOI: 10.4000/economierurale.4402

ISSN: 2105-2581

\section{Publisher}

Société Française d'Économie Rurale (SFER)

Printed version

Date of publication: 15 July 2014

Number of pages: 69-85

ISSN: 0013-0559

Electronic reference

Romain Melot, «Droits de propriété et d'usage sur la terre. Une étude statistique des recours contentieux en matière de fermage ", Économie rurale [En ligne], 342 I juillet-août 2014, mis en ligne le 15 juillet 2016, consulté le 21 décembre 2020. URL : http://journals.openedition.org/economierurale/ 4402 ; DOI : https://doi.org/10.4000/economierurale.4402 


\title{
Droits de propriété et d'usage sur la terre Une étude statistique des recours contentieux en matière de fermage
}

\author{
Romain MELOT• Inra-Sadapt, Paris
}

Dans le cadre des droits définis par le statut du fermage, les tribunaux des baux ruraux traitent des litiges opposant bailleurs et fermiers. L'étude présentée ici vise à analyser, à partir d'une enquête statistique portant sur environ 1500 jugements rendus durant une année en France, la nature des prétentions juridiques les plus fréquemment adressées aux juridictions et à comprendre les logiques de ces recours. L'auteur montre notamment que la majorité des recours de fermiers contestant le congé n'est pas liée aux conséquences financières de la rupture du bail, mais vise avant tout la poursuite de la relation contractuelle, dans le cas des projets de reprise par le bailleur. L'étude met également en évidence la place importante occupée par les demandes liées à la cession du bail et au droit de préemption.

MOTS-CLÉS : fermage, contentieux, droits de propriété, terres agricoles, conflits

\section{Property rights and using rights on farmland: a statistical study of litigation concerning farm tenancy}

In the legal framework of the "farm lease act", the French "agricultural rent tribunals" adjudicate disputes between landlords and tenants. Based on a statistical inquiry on about 1500 cases filed during one year in France, the study tries to analyse the nature of legal claims most usually exposed and to understand the logic of actions. We found that the majority of proceedings of tenants against leaves are not linked to the financial consequences of breach of contract, but aim above all to maintain the contractual relation, when landowners project to take the land back. The study the important role played by claims for assignment of lease or pre-emption rights. (JEL R52, Z13)

KEYWORDS: farm tenancy, litigation, property rights, farmland, conflicts

$\mathbf{L}^{\mathrm{s}}$ e statut du fermage et du métayage ${ }^{1}$ est généralement considéré comme garantissant une situation de grande stabilité au locataire exploitant, lequel, à la différence des locataires de baux d'habitation, dispose d'un droit au renouvellement de son bail. Conquête majeure des syndicats agricoles à la Libération, à la suite de l'échec d'un premier projet sous le Front populaire, l'obtention du statut visait à consacrer la libération du fermier de la tutelle oppressante d'un propriétaire disposant de droits importants sur la gestion foncière et

1. Nous utilisons par la suite l'expression consacrée «statut du fermage » par souci de concision. culturale des biens loués, la fixation des loyers, ainsi que sur les modalités de rupture du contrat.

Si la situation des exploitants locataires dans la France d'aujourd'hui n'est plus soumise à une logique de captation de la rente, la sécurité juridique offerte par le statut du fermage n'est pas totale. Le développement de la part des bailleurs de logiques patrimoniales en réponse à une pression foncière accrue sur les terres agricoles, mais aussi la concurrence entre agriculteurs pour l'accès au foncier sont deux phénomènes qui conduisent à s'interroger sur la stabilité de la relation contractuelle. Dans les situations où les litiges éclatent 
au grand jour sous la forme de recours judiciaires, l'intérêt d'une enquête sur le contentieux du bail rural consiste à traiter de ces enjeux dans une perspective de sociologie juridique, attentive aux formes de mobilisation du droit (Serverin, 2000). C'est ce que vise l'enquête présentée ici, menée à partir d'un panel d'affaires jugées par les tribunaux en France durant une année. S'inscrivant dans le cadre des analyses conduites en sciences sociales sur les dynamiques conflictuelles dans les espaces ruraux (Darly, Torre, 2008), elle tente d'expliquer la manière dont les preneurs cherchent à garantir la pérennité de leur exploitation dans un contexte judiciaire et, inversement, quelles sont les marges de manœuvre utilisées par les bailleurs pour gérer leur bien avec une relative souplesse en dépit des protections conférées à l'exploitant.

\section{Le statut du fermage : mise en perspective historique et dispositions principales}

Les rapports entre propriétaires et fermiers qui caractérisent le monde rural actuel sont les héritiers d'une série de transformations qui ont peu à peu façonné la structure foncière des exploitations agricoles françaises. Elles ont abouti à l'adoption d'un statut du fermage qui est fortement marqué par la référence à un ordre public protecteur à l'égard du fermier, mais qui laisse néanmoins subsister des prérogatives substantielles pour le bailleur. En outre, les transformations profondes du monde agricole depuis les années d'après-guerre se sont traduites par des modalités de recours au fermage profondément renouvelées. C'est précisément le fonctionnement de cette relation de bail que se propose d'interroger l'étude statistique du recours au juge paritaire présentée ici.

\section{Une évolution historique vers un rééquilibrage des rapports entre bailleurs et fermiers}

Jusqu'au point de rupture que constitue l'instauration du statut du fermage à la Libération, le système foncier français a en effet été longtemps marqué par une captation de la rente foncière par les propriétaires. Dominante en particulier dans le Nord et l'Ouest de la France, la grande propriété foncière impose jusqu'à la Seconde Guerre mondiale des loyers élevés aux agriculteurs fermiers (Coulomb, 1993).

Reprenant des dispositions d'un projet de loi avorté sous le Front populaire, ainsi que des prescriptions édictées par le régime de Vichy, la réforme instaurée par loi du 13 avril 1946 instituant le régime actuel des baux ruraux constitue la clef de voûte juridique de cette rupture historique, qui s'inscrit dans un mouvement général en Europe visant à renforcer les droits du fermier (Courleux, 2011). La principale innovation instaurée par la réforme de 1946 consiste à rattacher par défaut toute location de bien agricole au régime du fermage, alors que les représentants des propriétaires fonciers (en particulier au Sénat) s'étaient efforcés, depuis l'instauration du Code civil napoléonien et tout au long du XIX $^{\mathrm{e}}$ siècle, de réserver le fermage au cas spécifique des grands exploitants du bassin parisien. Ainsi, les exceptions au statut du fermage (le métayage, mais aussi et surtout les formes de location annuelle) demeuraient la forme la plus répandue de contractualisation. Le cadre juridique de référence était principalement fourni par les « usages locaux », le plus souvent issus d'un contexte social et historique très favorable au propriétaire (durées de location très courtes, définition restrictive des modalités d'indemnisation du locataire) (Aberdam, 1982). 


\section{Encadré 1. Rappel des principales dispositions du code rural relatives au statut du fermage}

Le bail rural est défini très largement comme une « mise à disposition à titre onéreux d'un immeuble à usage agricole [... » (art. L. 411-1 du Code rural). Cette définition d'une grande souplesse signifie qu'un exploitant peut revendiquer le bénéfice du statut du fermage à partir du moment où l'usage agricole est avéré et où la preuve d'un paiement du loyer est fournie, que le contrat soit écrit ou oral (dans la mesure où le même article dispose que « la preuve des contrats [...] peut être apportée par tous moyens [...]»).

Le bail rural est conclu pour une durée de neuf ans et le droit au renouvellement est énoncé explicitement. Les dispositions du Code rural encadrent strictement les conditions de rupture du bail, les motifs de congés admis étant énoncés de manière limitative. Il peut s'agir d'une faute du preneur se traduisant par un impayé ou une mauvaise gestion manifeste du bien loué ( « les agissements du preneur de nature à compromettre la bonne exploitation du fonds », art. L. 411-31 du code rural). Le changement de destination des terres louées, devenues constructibles en vertu d'une décision publique en matière de droit de l'urbanisme, entraîne également la possibilité de rompre le bail à tout moment (art. L. 411-32). Enfin, le bailleur est autorisé à refuser le renouvellement du bail s'il souhaite reprendre les terres louées pour lui--même, son conjoint ou ses descendants, à condition que la reprise soit liée à une activité agricole durant au moins neuf ans (art. L. 411-58).

À l'expiration du bail, le preneur sortant a droit à une indemnité pour les améliorations foncières et culturales qu'il a réalisées, « quelle que soit la cause qui a mis fin au bail » (art. L. 411-69). Le preneur est doté d'un droit de préemption sur la vente du bien loué et peut saisir le tribunal paritaire d'une demande en nullité de la vente ou de fixation du prix d'acquisition (art. L. 412-1). Le preneur dispose également de la faculté de demander au bailleur que son bail soit cédé à son conjoint ou ses descendants. À défaut d'accord du bailleur, il peut saisir le tribunal paritaire (art. L. 411-35).
À la stabilité que procure le droit au renouvellement du bail créé par le statut du fermage s'ajoute le droit reconnu au fermier, y compris en cas de faute de sa part ayant abouti à la rupture du bail, à récupérer son investissement sous la forme d'une compensation généralement dénommée sous le terme d' "indemnité de sortie », laquelle est due, quel que soit le motif du congé dès lors qu'une amélioration a été apportée aux biens loués par le fermier. Cette indemnité procure ainsi à l'exploitant une garantie financière qui lui permet de s'engager plus sereinement dans une stratégie d'investissement.

Le droit du bail rural tel que mis en place sous la forme moderne du statut du fermage est caractérisé par l'importance des limitations à la liberté contractuelle. Pour Joseph Hudault, cette limitation à la liberté contractuelle reflète un trait général du droit rural, à savoir la combinaison d'une « intervention de l'État, de la mise à l'écart de certaines règles du droit privé et du rôle joué par une réglementation d'ordre public » (Hudault, 2006).

Le caractère d'ordre public du statut du fermage (article L. 415-2 du code rural) a pour principal effet d'interdire le renoncement par avance à des droits non nés dans le cadre de clauses contractuelles ${ }^{2}$. En dépit de ces garanties, les évolutions suivies par le statut ont aménagé des prérogatives importantes au profit du bailleur, notamment en matière de résiliation. En premier lieu, un véritable « droit de résiliation » pour raison d'urbanisme a été reconnu au bailleur (loi du 30 décembre 1967), même

2. Cass. Soc., 24 avril 1952 : D. 1952, p. 721. 
si celui-ci a été restreint aux seules zones urbaines des documents d'urbanisme par la jurisprudence ${ }^{3}$, puis par des dispositions législatives (loi du 15 juillet 1975). En outre, en cas de changement de la destination des sols, la possibilité de conclure des conventions d'occupation précaire a été introduite (loi du 4 juillet 1980), dispositif dont l'encadrement est parfois problématique lorsqu'existent de fortes pressions spéculatives dans les zones périurbaines (Debeaurain, 2008).

Le droit de reprise du bailleur est encadré par des conditions précises (notamment l'obligation du propriétaire ou de son descendant d'exploiter les terres pendant une certaine durée). Cependant, dès lors que ces conditions sont remplies, les différentes évolutions législatives ont conservé le principe suivant lequel le droit de reprise au profit du bailleur ne devait pas être soumis à un contrôle d'opportunité a priori, à partir du moment où celui-ci ne compromet pas gravement l'exploitation du preneur (le contrôle a posteriori étant quant à lui peu usité) (Lemonnier, 2008).

\section{Transformations et impact du monde agricole actuel sur le recours au fermage}

Les pratiques contractuelles en matière de bail constituent un objet privilégié d'observation des usages sociaux du droit dans le monde agricole. En effet, les recherches sociologiques montrent le rôle central joué par l'intensité du recours au fermage, à l'intersection des pratiques matrimoniales et successorales, pour comprendre le profil des systèmes fonciers ruraux observables localement. Ces recherches soulignent de plus que l'intensité du recours au fermage correspond à une réalité complexe : les régions agricoles connaissant un taux de faire-valoir indirect important ne sont pas homogènes socialement (une tradition

3. Cass. $3^{\text {e }}$ civ., 14 mai 1974 : Bull. civ. 1974. III, $\mathrm{n}^{\circ} 189$. ancienne et liée à de grandes exploitations dans le Bassin parisien, une évolution plus récente en Bretagne et en Normandie) (Barthez, Fouquet, Villac, 1988).

Par ailleurs, l'appréciation de la place du fermage dans le monde agricole d'aujourd'hui est d'autant plus délicate que le modèle de l'agriculture familiale est en voie d'évolution. Sur la base d'une analyse approfondie des indicateurs statistiques sur l'évolution des exploitations, Jacques Rémy suggère ainsi l'émergence d'un modèle « post-familial », marqué notamment par la montée en puissance du fermage lié à des formes sociétaires (Rémy, 2013).

En outre, durant les trois décennies d'après-guerre s'affirme la montée en puissance de l'urbanisation et du développement à un rythme accéléré du phénomène de conversion des terres agricoles. Cette évolution peut amener les bailleurs qui anticipent ces conversions à faire pression auprès de leurs locataires pour qu'ils ne revendiquent pas le bénéfice du statut du fermage. Des enquêtes locales menées auprès de propriétaires fonciers et d'exploitants fermiers mettent en évidence la spécificité du contexte périurbain : les exploitants fermiers acceptent parfois de ne pas faire valoir leur droit (à la poursuite du bail et à l'indemnisation), afin de préserver leur réputation de «bon locataire » sur le marché local des bailleurs, ce qui correspond objectivement à une violation du statut (Alavoine-Mornas, Léger, 2013).

\section{Analyse statistique du contentieux et modalités de recours au juge paritaire}

La méthodologie d'analyse statistique utilisée ici s'inspire des travaux menés dans une perspective d'analyse empirique du contentieux, notamment par des juristes et des sociologues. La limite de cette méthode est qu'elle ne porte pas sur des enquêtes directes auprès des acteurs du procès : l'historique de la relation contractuelle et 


\section{Encadré 2. Méthodologie de l'enquête}

La présente recherche est la première enquête statistique réalisée au niveau national sur le contentieux des baux ruraux à partir d'un échantillon d'affaires. Elle est le fruit d'une convention d'étude entre l'Inra et le ministère de la Justice (sous-direction de la statistique et des études). Le travail de codage des décisions analysées dans le cadre de ce rapport a été réalisé par le Bureau de la collecte de la Sous-direction de la statistique et des études à Nantes, qui a été également associé à la conception de la méthodologie d'enquête. Le champ de l'enquête est constitué de l'ensemble des affaires jugées par les tribunaux paritaires des baux ruraux durant une période d'une année (du $1^{\text {er }}$ avril 2010 au $1^{\text {er }}$ avril 2011). Les tribunaux paritaires sont des juridictions constituées dans chaque tribunal d'instance par le juge d'instance et quatre assesseurs élus qui ne sont pas des magistrats professionnels (deux représentants des bailleurs et deux représentants des preneurs). Ils ont compétence pour juger des litiges liés au bail rural (en dernier ressort jusqu'à 4000 euros et à charge d'appel au-delà). L'échantillon définitif est constitué de 1478 jugements statuant sur le fond (les décisions avant dire droit ou constatant un abandon de procédure ayant été écartées, dans la mesure où elles ne permettent pas d'identifier précisément les prétentions des parties). Le traitement statistique a été réalisé au moyen d'une grille d'analyse d'une quarantaine de variables portant sur les caractéristiques générales des affaires, le type de contrat litigieux, la nature des biens loués, le profil des parties et de leurs prétentions juridiques (nature et objet des demandes, décision du tribunal). du litige ne peut donc être retracé que dans la limite des informations disponibles dans les jugements. Son intérêt en revanche est de pouvoir observer comme un phénomène social l'usage du tribunal, à partir d'échantillons représentatifs de l'activité des juridictions du fond, en proposant une analyse statistique des prétentions soulevées par les demandeurs.

Pour s'en tenir uniquement aux recherches menées en France ayant mis en œuvre une étude quantitative des recours sur les questions de bail, on peut se référer aux enquêtes très approfondies menées sur les litiges liés aux baux d'habitation à propos de la fixation des loyers (Beroujon, Bruxelles, 1993) et des procédures d'expulsion (Ancel, 2003). Pour le sujet qui nous intéresse ici, à savoir le bail rural, les travaux de référence ont été menés dans les années 1970 par Chantal de Crisenoy et Daniel Boscheron. En se basant sur des échantillons d'affaires locales, croisés avec des documents d'huissiers, les auteurs soulignent en particulier la place essentielle occupée par les litiges sur les reprises - enjeu sur lequel nous revenons plus loin (Boscheron, de Crisenoy, 1982 ; de Crisenoy, 1988).

\section{Les pratiques contractuelles au prisme du contentieux}

La position occupée par l'outil juridique qu'est le bail rural est essentielle sur le plan territorial, non seulement parce que les terres agricoles couvrent l'essentiel de la superficie du territoire national, mais aussi en raison de la place dominante du faire-valoir indirect dans l'agriculture française. Même si les baux ruraux ne font en eux-mêmes l'objet d'aucun enregistrement à visée statistique, il est possible de s'interroger sur les caractéristiques des biens litigieux en s'appuyant sur les données foncières et économiques en matière agricole. Ces considérations nous amènent à identifier la contribution respective des bailleurs et les fermiers aux saisines enregistrées par les juridictions paritaires. 


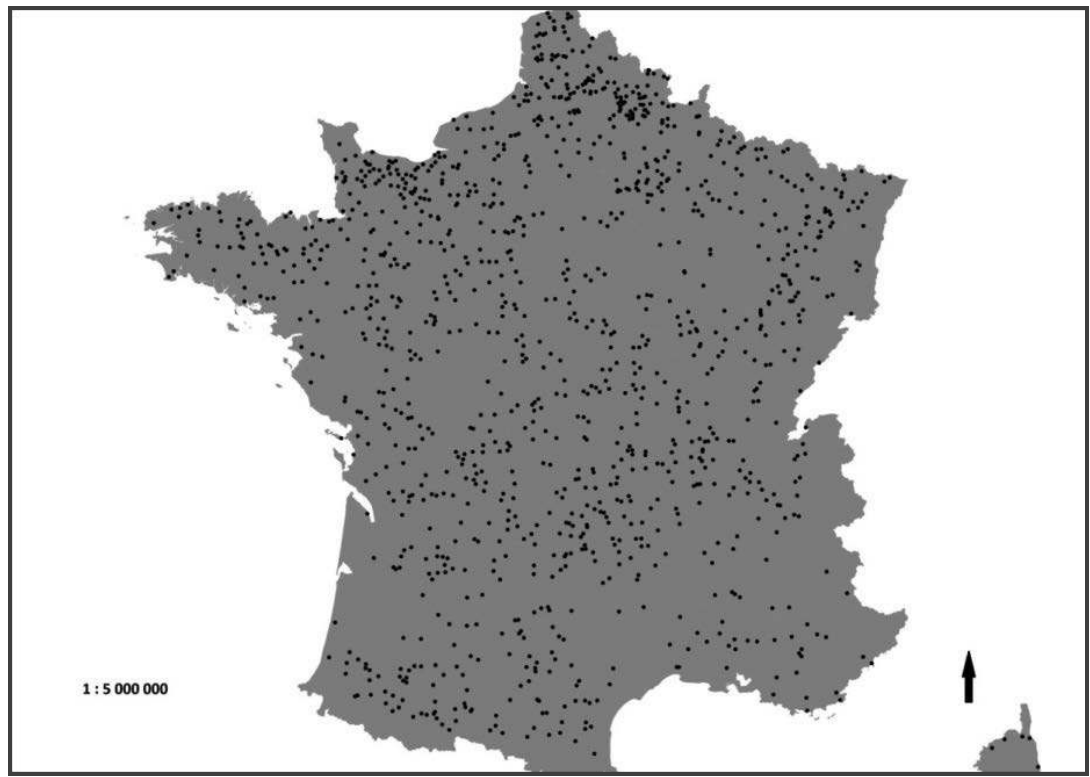

Source : l'auteur.

\section{Les caractéristiques des baux litigieux dans l'échantillon d'enquête}

Près des deux tiers $(61,7 \%)$ des surfaces agricoles étaient exploitées en 2010 par le biais du fermage auprès d'un tiers. Ce phénomène est dû notamment à la place de plus en plus importante occupée par la mise en location effectuée par des exploitants qui ne sont plus en activité (Courleux, 2011).

Bien que majoritaire au niveau national, le recours au fermage est très inégalement mis en œuvre suivant les régions et la localisation des affaires en matière de bail reflète largement cette diversité géographique et historique (carte 1). On retrouve en effet des différences entre des régions de petite propriété agricole (Provence) et les régions de grandes céréalicultures (Bassin parisien et Picardie) ainsi que la Normandie, dans lesquelles la tradition du fermage (et en particulier des « grands fermiers ») est ancrée de manière ancienne. On notera cependant un relatif évitement du tribunal en région parisienne, où le fermage est pourtant prédominant.

Les pratiques contractuelles en matière de bail rural, lesquelles ne font pas l'objet d'enquêtes systématiques ni d'enregistrement administratif, demeurent mal connues $^{4}$. Il est donc difficile de préciser en quoi les biens litigieux dont il est question dans l'échantillon d'affaires présentent des spécificités par rapport à l'ensemble des contrats existants dans ce domaine. Il est cependant possible de relever un certain nombre de caractéristiques des contrats concernés par les litiges étudiés,

4. L'analyse des données de la dernière grande enquête nationale sur le foncier agricole (Enquête foncière de 1992) met par ailleurs l'accent sur deux phénomènes d'importance (mais difficiles à appréhender dans le cadre de notre étude) concernant les baux ruraux : la multicontractualisation (le fait pour un fermier d'avoir plusieurs bailleurs différents) et le fermage intrafamilial (le fait pour un propriétaire de louer à bail ses terres au profit de son ou ses enfants) (Barthélémy, Fiche, 1994). 
qu'il s'agisse du profil des parties et des biens loués ${ }^{5}$.

Les baux qui font l'objet de litige dans notre échantillon d'enquête sont a priori marqués par leur stabilité, à la fois sur le plan de la relation contractuelle (en majorité, il s'agit de relations anciennes entre deux parties) et sur le plan du cadre juridique de référence (la plupart des demandeurs se réfèrent au statut du fermage). Cependant, cette tendance générale ne doit pas cacher le fait que, dans un nombre non négligeable d'affaires, l'appréciation de cette stabilité est plus contrastée.

Concernant la stabilité de la relation contractuelle en premier lieu, il convient de relever qu'il arrive que les baux changent de main, et ce des deux côtés (du fait du bailleur, comme du fait du preneur). Les mutations qui impliquent un changement de propriétaire concernent ainsi un peu plus du quart des baux litigieux (26\%). Certes, la relative fréquence des mutations s'explique aussi par la durée de la relation contractuelle en matière de bail rural, beaucoup plus longue que pour les baux d'habitations. Mais le fait que le propriétaire change depuis la signature du bail initial peut ainsi impliquer une modification de l'attitude dans la gestion du bien de la part des nouveaux bailleurs. Ces mutations font suite à une succession (15\% des affaires), une donation $(7 \%)$, un partage $(1 \%)$ ou une acquisition (3\%). Par ailleurs, les successions aboutissent parfois à une situation d'indivision (dans $11 \%$ des affaires) qui peut être source de divergences entre co-indivisaires sur les stratégies patrimoniales. D'autre part, la modification de la

5. De manière paradoxale, il est très fréquent que le texte des jugements ne fournisse que des indications très sommaires sur la nature de l'activité agricole pratiquée sur les terres objet du recours, dès lors que ces indications ne sont pas nécessaires à la solution du litige (dans $62 \%$ des affaires, cette information n'a pu être renseignée). relation contractuelle peut être aussi le fait du fermier. Il arrive ainsi (dans $14 \%$ des affaires) que les biens loués fassent l'objet de la part du fermier d'une mise à disposition (avec accord du bailleur ou simplement de fait), dans la quasi-totalité des cas sous la forme d'une cession. Ces situations expliquent, comme exposé plus loin, le profil de certains demandeurs (parmi lesquels figurent les bénéficiaires de cession) et la nature de certaines demandes (visant l'autorisation d'une cession de bail par le tribunal).

En second lieu, le fait que le cadre juridique apparaisse comme stable du point de vue de la référence, très majoritaire, au statut du fermage, n'exclut pas qu'une proportion importante d'affaires s'inscrivent dans un contexte d'incertitude des droits. En effet, l'incertitude est plus souvent l'objet d'un litige sur l'existence du contrat que sur sa nature. Lorsqu'ils saisissent la juridiction paritaire, les preneurs se situent ainsi en très grande majorité dans une relation contractuelle qu'ils qualifient de bail rural, la plupart du temps sous la forme la plus commune du bail à ferme (soit $85 \%$ des affaires, si l'on considère qu'en l'absence d'indications, il est probable que le contrat soit de cette nature). Les autres cas renvoient à des formes de contrat qui offrent des droits équivalents, voire plus importants, ou correspondent à des spéculations particulières comme la viticulture (bail à long terme, métayage, bail à complant, bail à colonat partiaire), tandis que les références à d'autres cadres contractuels sont beaucoup plus rares (vente d'herbe, bail d'habitation, bail commercial...). En revanche, dans une affaire sur cinq environ (19\%), il n'est pas possible de relever la mention d'une date de contrat, soit que le texte du jugement soit muet sur cette information, soit que cette date ne soit pas connue précisément du demandeur en l'absence d'écrit. L'absence d'une date de contrat est une indication du faible degré de formalisation 
et de l'incertitude quant à l'existence du bail ${ }^{6}$.

Par conséquent, l'analyse du profil des baux enquêtés laisse apparaître des caractéristiques propres à la relation contractuelle ou à l'historique du contrat qui peuvent se révéler propices à la naissance de litiges. Mais les dynamiques de recours au tribunal ne prennent tout leur sens que dans la mesure où l'action en justice est resituée par rapport aux droits distincts mobilisés par chacune des parties contractantes.

\section{Les auteurs de recours : les fermiers jouent un rôle actif}

Alors que dans le contentieux des baux d'habitation, l'essentiel des recours sont le fait de bailleurs, la situation est totalement différente dans le cas du contentieux des baux ruraux, puisque les affaires initiées par les preneurs sont majoritaires (53\%) face aux recours engagés par les bailleurs $(43 \%)$. Notons par ailleurs que les cocontractants au bail ne sont pas les seuls protagonistes des affaires. Les demandeurs peuvent être également des personnes revendiquant le bénéfice d'une cession de bail (dans $2 \%$ des affaires, mais dans ce cas, sauf quelques exceptions, ils n'agissent qu'en complément d'une action du preneur), ou encore les tiers acquéreurs (moins de $1 \%$ ) concernés par la mise en œuvre du droit de préemption du preneur. Enfin, d'autres tiers (1\% des demandeurs), anciens propriétaires ou anciens bailleurs (par rapport au bail litigieux), peuvent être auteurs des recours.

6. L'incertitude sur l'existence du bail n'est pas liée au caractère verbal du contrat, lequel n'est pas un obstacle lorsqu'il s'agit de dater la conclusion du bail. Dans les affaires concernant des baux verbaux ( $15 \%$ de l'échantillon), la date de conclusion est majoritairement mentionnée (62\% de ces affaires). Il est vrai qu'à côté de la rédaction d'un bail écrit, existent d'autres moyens de formalisation de la relation contractuelle. Par ailleurs, les affaires dans lesquelles le contrat est formalisé par un acte notarié constituent une minorité (27\%).
Situer le litige dans le cadre de la relation contractuelle constitue un préalable nécessaire pour identifier les logiques de recours au tribunal. Le contentieux du bail, caractéristique en cela des recours en matière contractuelle, est en effet très souvent un contentieux de la résiliation. Pour bien comprendre le contexte dans lequel interviennent les affaires, il est donc important de savoir si un congé est déjà intervenu et si c'est le cas, quel a été le motif invoqué par la partie à l'initiative de la rupture, sachant qu'à de rares exceptions près il s'agit de la partie bailleresse ${ }^{7}$. Il apparaît alors que les affaires initiées par les bailleurs et celles engagées par les preneurs se situent sur un plan radicalement différent si l'on s'en tient au seul critère des modalités du congé.

Les situations dans lesquelles les bailleurs ne saisissent le tribunal qu'après avoir délivré un congé ne représentent qu'un recours sur trois (29\%). En effet, très souvent ces derniers prennent l'initiative d'aller en justice pour faire prononcer directement par le tribunal la résiliation du bail, sans délivrer eux-mêmes le congé. Par ailleurs, dans d'autres affaires également, le propriétaire n'a pas délivré de congé, car il ne reconnaît pas l'existence d'un contrat et ne se considère donc pas comme bailleur. Il demande alors au tribunal de constater que son bien fait l'objet d'une occupation sans titre. Enfin, le litige peut porter sur les modalités d'exécution d'un contrat en cours (fixation du montant du fermage). Mais, comme nous le verrons plus loin, c'est avant tout dans un contexte de rupture de la relation contractuelle (ou de contestation de son existence) que s'inscrivent majoritairement les litiges.

7. Le congé n'est que très rarement délivré à l'initiative du preneur. Seule une petite minorité d'affaires (28 soit 1,8\% de l'échantillon d'enquête) est concernée par ce cas de figure, le motif le plus fréquemment évoqué étant alors le départ en retraite du preneur. 
Les fermiers sont à l'inverse plus nombreux à agir en justice à la suite d'un congé que leur a notifié le bailleur (53\%). Une part importante de leurs recours intervient néanmoins alors qu'aucun congé ne leur a été notifié. Un élément d'explication réside là encore dans le fait que le litige peut porter, comme dans le cas des recours initiés par les bailleurs, sur l'existence même du bail. Une demande visant à bénéficier du statut du fermage ne peut ainsi faire suite à un congé, dans la mesure où le bail à ferme n'est pas reconnu par le propriétaire. En outre, un certain nombre de demandes présentées par le preneur ne sont pas la conséquence d'une rupture du contrat. Il s'agit notamment des demandes de cession du bail ou de celles liées au droit de préemption, sur lesquelles nous reviendrons.

L'analyse des motifs de congé permet de préciser le profil des affaires qui interviennent alors que la rupture de la relation contractuelle (au sens juridique) est déjà consommée.

Lorsque les bailleurs sont les auteurs des recours, c'est très souvent dans le cadre d'un schéma fréquemment rencontré dans le cadre du contentieux des contrats en général, celui de l'impayé. Le défaut de paiement des fermages est en effet le premier motif de congé invoqué (dans $49 \%$ des affaires où le motif de congé est connu $)^{8}$. Il apparaît ainsi que, souvent, l'incitation première des bailleurs à saisir la juridiction des baux ruraux n'est pas tant de faire valider un congé dont la motivation est susceptible de prêter à discussion, mais plutôt d'obtenir la liquidation d'une créance. Le tribunal est alors davantage sollicité pour faire pression sur un débiteur récalcitrant et obtenir un titre exécutoire que pour trancher un litige concernant des droits incertains ou contestés.

8. Dans les affaires faisant suite à un congé, le motif de ce congé n'a pu être renseigné que dans $68,6 \%$ des cas pour ce qui est des recours de bailleurs et dans $55 \%$ des cas pour ce qui est des recours de preneurs.
Notons également que parmi les autres motifs de congé que le défaut de paiement, celui de mauvaise exploitation du fonds, mentionné en deuxième position (15\%), est lui aussi généralement lié à des demandes financières, puisqu'il peut justifier de la part du bailleur une demande d'indemnité pour dégradations. Les autres cas admis par la législation comme motifs légitimes de rupture du bail sont moins fréquents : reprise des terres par le bailleur pour son compte ou pour le compte de ses proches $(14 \%)$, rupture du contrat en raison d'une cession de bail non autorisée (12\%), congé à la suite d'un changement de destination des terres louées devenues constructibles (4\%) (tableau 1).

La situation est très différente dans le cas des preneurs puisque les recours qu'ils engagent lorsque le bail a été rompu par le bailleur interviennent très majoritairement à la suite d'un congé pour reprise $(72 \%$ des motifs de congés connus). Ces résultats confirment l'enjeu central des contestations sur les reprises que soulignaient les études antérieures (citées plus haut) à propos des comportements de saisine des fermiers (Boscheron, de Crisenoy, 1982). Beaucoup plus modeste est la part occupée par les autres motifs de congé comme le changement de destination des terres louées $(11 \%)$, la mauvaise exploitation du fonds (8\%), le défaut de paiement (4\%) ou encore la cession du bail sans autorisation $(1 \%)$. En définitive, sur la totalité des recours contentieux engagés par les fermiers, au moins $20 \%$ sont des recours à la suite d'un congé pour reprise9 (tableau 2).

La rareté des recours contre des congés motivés par une mauvaise exploitation du fonds trouve sans doute sa source dans le comportement des bailleurs « en amont », c'est-à-dire dans l'usage limité qu'ont ces

9. Sans doute davantage, dans la mesure où l'information sur les motifs de congé n'est pas toujours mentionnée dans le texte des jugements, comme indiqué plus haut. 
Droits de propriété et d'usage sur la terre

Tableau 1. Motifs de congé dans les affaires initiées par le bailleur

\begin{tabular}{lcc}
\hline \multicolumn{1}{c}{ Motif de congé } & Nombre d'affaires & $\%$ \\
\hline Défaut de paiement des fermages & 63 & 49,6 \\
Mauvaise exploitation du fonds & 20 & 15,7 \\
Reprise des biens loués dont... & 18 & 14,1 \\
Reprise pour son propre compte & 10 & 7,8 \\
Reprise pour le compte d'un descendant & 3 & 2,3 \\
Autre cas de reprise ou motif de reprise indéterminé & 5 & 3,9 \\
Cession du bail dans des conditions illégales & 16 & 12,5 \\
Changement de destination des terres louées & 6 & 4,7 \\
Défaut d'autorisation administrative (capacité, contrôle des structures) & 4 & 3,1 \\
Total & 127 & 100 \\
\hline
\end{tabular}

Source : l'auteur.

Tableau 2. Motifs de congé dans les affaires initiées par le preneur

\begin{tabular}{lcc}
\hline \multicolumn{1}{c}{ Motif de congé } & Nombre d'affaires $(\mathbf{n = 2 1 5 )}$ & $\%$ \\
\hline Reprise des terres louées dont... & 156 & 72,5 \\
Reprise par le bailleur pour son propre compte & 76 & 35,3 \\
Reprise par le bailleur pour le compte d'un descendant & 59 & 27,4 \\
Motif de reprise indéterminé & 21 & 9,7 \\
Changement de destination des terres louées & 25 & 11,6 \\
Mauvaise exploitation du fonds & 18 & 8,3 \\
Défaut de paiement des fermages & 10 & 4,6 \\
Cession du bail dans des conditions illégales & 4 & 1,8 \\
Défaut d'autorisation administrative (capacité, contrôle des structures) & 2 & 0,9 \\
\hline
\end{tabular}

Source : I'auteur.

derniers de ce motif, dont la preuve peut être délicate. À l'inverse, c'est plutôt le comportement «en aval» des preneurs qui explique leur réticence à agir en justice pour remettre en cause des motifs de congé dont la discussion ne donne que peu de prise à interprétation, comme les congés pour impayé de loyers. Les affaires initiées par les preneurs à la suite d'un congé pour reprise se situent en quelque sorte dans un entre-deux. Un tel motif de congé offre une certaine latitude d'argumentation pour le bailleur, tout en laissant la possibilité d'une contestation par le preneur, lequel peut défendre la nécessité de pérenniser son activité.

Plus fondamentalement, l'importance des litiges sur les reprises de terres (qui peuvent inclure également des reprises de bâtiments) renvoie à deux types d'enjeux économiques et sociaux : la plus-value foncière opérée sur des terres agricoles destinées à la construction et la pérennité de l'exploitation agricole comme unité économique.

Concernant le premier point, la question de la réalisation de plus-values foncières intervient explicitement dans le cas du changement de destination des terres louées, mais peut se poser aussi de manière implicite dans le cas des reprises.

En effet, un projet de reprise peut dissimuler une visée patrimoniale dans le cas où le bailleur, qui ne bénéficie pas d'une décision d'urbanisme rendant ses terrains 
constructibles, anticipe leur changement de destination futur. Dans ce cas de figure, congédier un exploitant pour laisser des terrains en friche et faciliter ainsi leur changement de destination est une stratégie observée au niveau local, stratégie qui repose souvent sur la renonciation implicite du fermier à exercer un contrôle a priori sur le congé ou a posteriori sur l'effectivité du projet de reprise (Alavoine-Mornas, Léger, 2013 ; Guéringer, 2013). L'atout que peut constituer le droit de reprise est de permettre la mise en œuvre d'une stratégie d'anticipation ou plutôt d'une stratégie d'attente dans l'optique d'une évolution espérée des règles d'urbanisme. Il n'est pas possible d'évaluer précisément la place occupée par ces stratégies dans les choix faits par les bailleurs de reprendre les biens loués. Mais il est à noter que dans quelques affaires, des preneurs à l'origine de recours appuient explicitement leur argumentation sur le caractère économiquement fictif de projets de reprise invoqués par leurs bailleurs.

Un certain nombre d'arguments laissent cependant penser que ce phénomène demeure sans doute limité. En premier lieu, de telles stratégies d'anticipation foncière semblent davantage s'appliquer aux communes périurbaines dans lesquelles les terres agricoles sont soumises à une pression importante, ce que suggèrent les enquêtes locales réalisées sur le sujet auprès de propriétaires fonciers et d'exploitants agricoles. Par ailleurs, dans les affaires engagées par les preneurs à la suite d'un congé pour reprise, il est fréquent de trouver des terres louées dont la surface est relativement importante (45\% des surfaces mentionnées dans ce type de recours sont supérieures à dix hectares et $24 \%$ supérieures à vingt hectares). Or, lorsque la reprise des terres louées porte sur des grandes surfaces, elle semble moins susceptible d'être liée à un projet de construction spéculatif, mais relever davantage d'une reprise inhérente à un projet agricole porté par un propriétaire exploitant.
Quelles que soient les motivations profondes des bailleurs, l'analyse des conditions dans lesquelles le contrat est rompu avant le recours judiciaire lui-même amène une série de questions. Dans le cadre des contestations portées à l'encontre des congés délivrés par les bailleurs, les prétentions des fermiers sont-elles essentiellement de nature indemnitaire ? Quelle est par ailleurs la nature des demandes soumises au juge lorsque le litige n'est pas la suite immédiate d'une rupture de bail ? Ces interrogations nous amènent à traiter du profil des demandes adressées aux juridictions dans le cadre du contentieux du bail rural.

\section{Solder la relation contractuelle ou préserver un usage ?}

L'analyse des demandes adressées aux tribunaux paritaires tend à montrer un spectre de prétentions juridiques très contrasté selon que l'action en justice est engagée par le bailleur ou par le preneur. Pour dresser grossièrement ce tableau, on peut opposer un bloc de prétentions relativement homogènes chez le bailleur, lesquelles s'organisent principalement autour du cadre (classique en matière contractuelle) de l'impayé, à un spectre beaucoup plus varié de revendications du côté du preneur, qui renvoie aux différents droits spécifiques dont il est doté dans une visée protectrice.

\section{Recours de bailleurs ciblés sur les conséquences de la rupture du bail}

Du côté du bailleur, deux motivations principales sont susceptibles de l'amener à saisir le tribunal : récupérer une créance et obtenir la fin d'une occupation (qu'il juge) illicite. À cet égard, le fait que la demande la plus fréquemment soumise aux juges ait pour objet la résiliation du bail (dans $62 \%$ des affaires initiées par un bailleur) doit être correctement interprété. Les bailleurs n'ont pas nécessairement d'intérêt pratique 
à solliciter la justice dans le seul but de faire prononcer judiciairement la résiliation $\mathrm{du}$ contrat, dans la mesure où ils ont tout loisir de délivrer eux-mêmes un congé à partir du moment où ils l'estiment motivé. Les enquêtes réalisées sur le contentieux du bail et de l'expulsion dans le domaine des baux d'habitation aboutissent à ce constat similaire que les demandes de résiliation judiciaire soumises par les bailleurs n'ont de sens qu'associées à d'autres demandes qui en sont les corollaires. La preuve en est que dans notre échantillon, les recours de bailleurs ayant pour objet la seule résiliation sont rares. En règle générale, les recours contentieux des bailleurs sont motivés soit par le souci de parvenir à la liquidation d'une créance que l'autre partie refuse de payer (les demandes d'arriérés de loyer concernent $36 \%$ des affaires initiées par les bailleurs), soit d'obtenir la garantie de l'intervention in fine de la force publique pour contraindre l'occupant à libérer les lieux (dans $39 \%$ des affaires, une demande d'expulsion est formulée). En bref, des effets pratiques que le bailleur ne peut obtenir par lui-même.

Les recours contentieux des bailleurs s'organisent donc de manière binaire autour d'un couple de prétentions associant soit généralement demande de résiliation et demande d'arriérés de loyers, soit demande de résiliation et demande d'expulsion. En effet, lorsqu'ils sont en position de demandeurs, les bailleurs qui ne saisissent le tribunal qu'aux seules fins de faire prononcer judiciairement la résiliation du bail sont ainsi relativement peu nombreux (10\% environ). D'une manière générale, ils ont tendance à soumettre au tribunal une série de demandes, puisque la proportion des bailleurs demandeurs se contentant d'une demande isolée ne dépasse pas le quart d'entre eux $(27 \%)$.

D'autres prétentions rejoignent dans une large mesure ces deux catégories de demandes. Il s'agit par exemple des demandes d'indemnité qui s'associent souvent aux demandes de paiement des loyers, soit qu'elles visent à faire indemniser des dégradations de la propriété (dans $15 \%$ des affaires, une indemnité pour dégradations est demandée en lien avec une mauvaise exploitation du fonds ou des constructions illicites), soit qu'elles revendiquent de manière plus floue un préjudice moral ou matériel ouvrant droit à dommages-intérêts (dans $10 \%$ des affaires se retrouvent des demandes de dommagesintérêts réparant un préjudice qui n'est pas toujours clairement identifiable d'après le texte du jugement). Il peut s'agir également de corollaires aux demandes d'expulsion, comme la revendication d'une indemnité d'occupation due à l'échéance du terme contractuel, dès lors que le preneur en place est devenu un occupant sans titre (dans $18 \%$ des affaires). A contrario, peu de demandes visent à ce que le tribunal paritaire se prononce sur l'interprétation des termes du contrat (c'est le cas des recours pour fixation du montant du fermage, qui ne représentent que $6 \%$ des actions engagées par les bailleurs). Il faut y voir le signe que lorsque les bailleurs décident d'agir en justice, c'est avant tout dans un contexte « post-contractuel». La relation contractuelle est déjà terminée et ils souhaitent voir l'institution judiciaire se prononcer sur les conséquences de la rupture du bail (tableau 3).

Les actions en justice des bailleurs sont caractérisées par un taux de réussite élevé. Dans les affaires où ils sollicitent la résiliation judiciaire du bail, les tribunaux font droit à $60 \%$ à leurs demandes. Ce taux de réussite atteint $75 \%$ dans le cas des demandes d'expulsion. Les demandes d'exécution provisoire rencontrent également un écho favorable à $57 \%$. Pour ce qui est des demandes à caractère financier, les demandes d'arriérés de loyer sont également majoritairement satisfaites par les tribunaux (à $88 \%$, dont $68 \%$ en totalité). De ce point de vue, le contentieux du bail rural ne se distingue pas radicalement du 
contentieux des baux d'habitation, mais aussi des autres contentieux contractuels : les demandes d'impayé y sont généralement caractérisées par un taux de satisfaction très élevé. Cependant, il est important de noter que les montants concernés apparaissent comme relativement modestes, notamment par rapport aux demandes indemnitaires formulées par les preneurs comme indiqué plus bas (le montant médian des demandes de paiement de fermage est de 3500 euros environ, le troisième quartile est de 7500 euros environ). Les demandes d'indemnité d'occupation sont concernées également par un taux de réussite important (satisfaites dans $72 \%$ des cas, dont $44 \%$ en totalité), mais correspondent à des montants encore plus modestes (médiane de 1400 euros environ). Les demandes d'indemnité pour dégradations représentent des montants plus importants (montant médian de 4000 euros environ et troisième quartile égal à 12500 euros environ), mais elles sont beaucoup moins souvent satisfaites par les tribunaux (dans $44 \%$ des cas seulement, dont uniquement $10 \%$ de satisfaction totale).
La situation est de nature très différente dans le cas des recours des fermiers. Le contexte et les motivations des actions en justice initiées par les fermiers sont beaucoup plus contrastés, comme nous l'indiquions plus haut. Une part importante des recours qu'ils initient a pour finalité la poursuite ou la reconnaissance de la relation contractuelle, alors que les bailleurs vont au tribunal pour organiser les conséquences de la rupture de cette relation. Il faut dire que le fermier est par nature la partie ayant le plus à perdre de la fin du bail.

À la différence des actions engagées par les bailleurs qui se focalisent sur deux ou trois éléments récurrents (l'impayé, l'expulsion), les recours des preneurs donnent lieu à un spectre de demandes beaucoup plus large et aussi beaucoup plus éclaté. Les demandes de poursuite du bail sont présentes dans $40 \%$ des affaires et constituent le versant du côté du preneur des demandes de résiliation du bailleur. Mais en dehors de cette demande très récurrente, aucune autre n'est présente dans plus de $17 \%$ des recours.

\section{Tableau 3. Demandes soumises aux tribunaux paritaires par les bailleurs ${ }^{10}$}

\begin{tabular}{lcc}
\hline \multicolumn{1}{c}{ Objet de la demande du bailleur à l'origine du recours } & Nombre d'affaires $(\mathbf{n}=\mathbf{6 3 4})$ & $\%$ \\
\hline Résiliation du bail & 397 & 62,6 \\
Expulsion & 253 & 39,9 \\
Fixation des arriérés de loyer & 233 & 36,8 \\
Exécution provisoire & 173 & 27,2 \\
Indemnité pour occupation & 118 & 18,6 \\
Indemnité due au bailleur pour dégradations & 98 & 15,5 \\
Demande de dommages-intérêts (préjudice indéterminé) & 69 & 10,9 \\
Montant du fermage & 43 & 6,8 \\
Expertise & 40 & 6,3 \\
Demande visant à faire constater l'occupation sans droit ni titre & 31 & 4,9 \\
Exécution de travaux & 29 & 4,6 \\
\hline
\end{tabular}

Source : I'auteur.

$10 \mathrm{Ne}$ sont indiquées ici que les demandes dont la fréquence est supérieure à $4 \%$ des affaires. 


\section{Les stratégies judiciaires des fermiers pour conserver l'usage des terres}

Pourquoi une telle dispersion au niveau des prétentions soulevées par les fermiers ? Deux éléments d'explication peuvent être apportés.

D'une part, les demandes des preneurs sont à la fois de nature indemnitaire et d'une autre nature. Autrement dit, alors que l'impayé est au cœur des logiques de recours chez les bailleurs, les demandes indemnitaires occupent une place certes importante, mais qui n'est pas centrale dans les actions engagées par les fermiers. Les indemnités dues au preneur sortant pour améliorations foncières et culturales (indemnités de sortie) ne sont présentes que dans environ $13 \%$ des recours ${ }^{11}$.

D'autre part, pour tenter de maintenir la stabilité de leur situation juridique, les fermiers mettent à profit une palette de droits très variés. L'objectif de pérennisation de la relation contractuelle passe donc par l'utilisation de nombreux outils juridiques liés au statut du fermage, outils qui sont mobilisés dans des proportions inégales.

La contestation des congés délivrés par les bailleurs est le premier chef de demande soulevé par les preneurs, comme indiqué plus haut (40\% des recours). Mais en deuxième position interviennent des recours qui ne se situent pas dans le contexte d'une rupture de contrat. Il s'agit des demandes visant à faire reconnaître par le juge l'existence même d'un bail rural (17\%). Comme nous l'avons vu, ce type de litige est lié avant tout à un désaccord sur l'existence d'une relation contractuelle et dans une moindre mesure seulement à un litige sur

11 Cependant, il est vrai qu'un certain nombre de demandes désignées de manière indéterminée comme des «dommages-intérêts » dans le texte des jugements, renvoient sans doute au préjudice réparé par ses indemnités de sortie. la nature ou le contenu de ce contrat ${ }^{12}$. Autrement dit, le choix d'aller au tribunal pour bénéficier du statut du fermage et non pour trancher un litige sur l'exécution du contrat ou les conséquences de sa rupture occupe une place non négligeable parmi les motivations qui incitent le fermier à saisir le juge. À cet égard, la protection offerte par la législation, qui assimile de manière très large toute mise à disposition de terrains agricoles au statut du fermage semble être particulièrement mobilisée par les exploitants. La qualification de bail rural est en effet de droit si la preuve d'un usage agricole et de la rémunération d'un loyer est apportée.

Mais d'autres ressources sont également mobilisées par les fermiers pour maintenir la jouissance des terres. La faculté dont ils disposent, en cas d'opposition du bailleur, de pouvoir demander au tribunal d'autoriser la cession du bail au profit d'un proche constitue ainsi une composante importante de la stabilité juridique offerte statut du fermage. Cette stabilité se poursuit alors au-delà même de l'horizon individuel de l'exploitant, puisqu'elle permet d'assurer la pérennité d'une unité économique, celle de l'exploitation agricole. La transmission de l'exploitation dans un cadre familial s'appuie dans ce cas de manière centrale sur le statut du fermage. Ce droit est mobilisé dans $12 \%$ des actions engagées par les fermiers, soit presque autant que le droit à indemnisation.

Enfin, une dernière manière, autrement plus radicale, de pérenniser la jouissance d'un bien exploité à des fins agricoles est

12 Nous considérons qu'il y a litige sur l'existence du contrat lorsqu'une partie (en l'occurrence le propriétaire des terres) conteste (de bonne foi ou non) tout lien d'obligation. Il y a litige sur la nature du contrat, lorsque le propriétaire reconnaît avoir un lien contractuel, mais considère, par exemple, que le statut du fermage ne s'applique pas (exemple : prêt à titre gratuit). 
l'acquisition en propriété. La saisine du tribunal pour mise en œuvre du droit de préemption concerne $8 \%$ des recours. Dans le cas des baux d'habitation, la vente du bien loué ouvre aussi un droit de préemption, mais constitue en soi un motif légal de congé. Tel n'est pas le cas concernant le statut du fermage, puisque le bail rural est censé se continuer avec le nouveau propriétaire si aucun congé n'a été délivré pour un autre motif (tableau 4).

Il est donc possible de parler d'une configuration originale, allant de la saisine pour qualification du contrat aux demandes de cession de bail. La revendication d'un droit de préemption correspond à une situation spécifique, dans la mesure où la décision du fermier de préempter le bien se traduit par une résiliation du bail. Cette configuration de litiges caractérise un profil de recours spécifique aux fermiers.

Les preneurs en position de demandeurs sont deux fois plus nombreux que les bailleurs à soumettre au tribunal des demandes uniques (ils sont $49 \%$ dans ce cas) et ils sont surtout très nombreux à ne demander que la poursuite ou le renouvellement du bail (23\%), sans assortir notamment ce type de recours de demandes indemnitaires. Ils sont également $5 \%$ à ne soumettre qu'une demande de qualification de bail rural et $4 \%$ à demander uniquement une cession de bail. Il est donc possible de distinguer deux profils de preneurs agissant en justice : d'une part ceux qui ne saisissent le tribunal que dans la seule intention de contester le congé et, d'autre part, ceux qui initient une démarche contentieuse plus complexe, impliquant la défense d'un faisceau de droits.

Dans les cas où existe un litige sur l'existence ou la nature du contrat, les recours du preneur portent naturellement très fréquemment sur des demandes de qualification du bail rural, mais sont aussi souvent dirigés autour de demandes visant à indemniser le trouble de jouissance $(18 \%)$ ou des « dommages-intérêts » $(17 \%)$. Du côté du bailleur, ce sont les demandes d'expulsion $(60 \%)$ et celles visant à faire constater une occupation sans titre (34\%) et l'indemnisation de cette occupation (14\%) ou des dommages-intérêts (14\%) qui sont parmi les plus courantes.

$$
\begin{gathered}
* \\
* *
\end{gathered}
$$

L'analyse des demandes soumises aux tribunaux paritaires met en évidence des

Tableau 4. Demandes soumises aux tribunaux paritaires par le preneur à l'origine du recours

\begin{tabular}{lcc}
\hline \multicolumn{1}{c}{ Objet de la demande du preneur à l'origine du recours } & Nombre d'affaires $(\mathbf{n}=\mathbf{7 8 1})$ & $\%$ \\
\hline Poursuite ou renouvellement du bail & 317 & 40,6 \\
Demande visant à faire qualifier le contrat de bail rural & 134 & 17,2 \\
Indemnité due au preneur sortant & 103 & 13,2 \\
Exécution provisoire & 95 & 12,1 \\
Cession du bail & 94 & 12,0 \\
Expertise & 86 & 11,0 \\
Indemnité pour trouble de jouissance & 71 & 9,1 \\
Exercice du droit de préemption du preneur & 63 & 8,0 \\
Demande de dommages-intérêts (préjudice non précisé) & 61 & 7,8 \\
Montant du fermage & 60 & 7,7 \\
Exécution de travaux & 37 & 4,7 \\
Demande visant à faire cesser un trouble de jouissance & 34 & 4,4 \\
\hline
\end{tabular}


logiques de recours contentieux très différentes suivant que le demandeur à l'origine de l'action en justice est un bailleur ou un preneur. Les recours des bailleurs ont avant tout pour objet d'organiser les conséquences de la rupture, qu'elles soient financières ou qu'elles visent à obtenir un titre exécutoire. Les logiques de recours des preneurs sont en revanche de nature distincte. Une part de leurs actions vise à contester le congé qui leur a été délivré en l'assortissant de demandes indemnitaires. Celles-ci bénéficient d'un taux de réussite plus mitigé que dans le cas des bailleurs, mais elles concernent également des enjeux financiers beaucoup plus importants.

Cependant, la majorité de leurs recours en contestation des congés ne sont pas liés aux conséquences financières de la rupture du bail, mais visent avant tout la poursuite de la relation contractuelle. Dans ce contexte, ils remettent avant tout en cause des projets de reprise des terres par le bailleur (pour son compte ou celui d'un conjoint ou descendant). Enfin, l'enseignement le plus intéressant à noter est qu'une proportion importante de leurs actions vise la poursuite de l'exploitation des terres louées alors même que le litige ne se déroule pas dans un contexte de rupture du contrat. Soit que c'est l'enjeu même de l'existence ou de la nature du bail qui est posé (demande visant à bénéficier du statut du fermage). Soit que la question de la sécurité juridique passe par la mobilisation d'autres droits, comme l'acquisition en propriété par le biais de la préemption ou la cession du bail à un conjoint ou un descendant. Sur ce dernier point, le contentieux entre preneur et bailleur constitue sans doute l'expression visible d'autres litiges liés à la concurrence entre exploitants agricoles pour l'accès à des terres en propriété, que cette concurrence porte sur l'acquisition en propriété (dans le cas des litiges sur l'exercice du droit de préemption) ou sur l'accès à des terres en location (lorsqu'est en jeu la cessibilité du bail).

\section{RÉFÉRENCES BIBLIOGRAPHIQUES}

Aberdam S. (1982). Aux origines du code rural. 1789-1900 : un siècle de débat. Nantes, INRA, 119 p.

Alavoine-Mornas F., Léger C. (2013). Les logiques foncières des agriculteurs dans un contexte périurbain : subir ou réagir ? In Bertrand N. (dir.), Terres agricoles périurbaines : une gouvernance en construction, Paris, Quae, p. 199-218.

Ancel P. (dir.) (2003). Les décisions d'expulsion d'occupants sans droit ni titre. Connaissance empirique d'un contentieux hétérogène. Rapport au ministère de la Justice.

Barthélémy D., Fiche D. (1994). Propriété foncière et exploitation agricole en 1992. Agreste Études, $\mathrm{n}^{\circ} 27,91$ p.
Barthez A., Fouquet A., Villac M. (1988). La structure foncière des exploitations. Prédominance du patrimoine au Sud, des exploitations au Nord. Économie et statistique, $\mathrm{n}^{\circ} 214$, octobre, p. 41-52.

Beroujon C., Bruxelles S. (1993). Règles juridiques, catégories statistiques et actions sociales. Droit et société, $\mathrm{n}^{\circ} 25$, p. 369394.

Boscheron D., de Crisenoy C. (1982). Statut des baux ruraux et contrôle des structures. Du droit à la réalité. Bordeaux, Presses de l'École nationale de la magistrature, $71 \mathrm{p}$.

Bué V. (2009). Ordre public et statut du fermage : entre protection et liberté. Droit rural, $\mathrm{n}^{\circ} 369$, dossier 12 . 
Coulomb P. (1993). De la terre à l'État. Droit de propriété, théories économiques, politiques foncières. Options méditerranéennes, $\mathrm{n}^{\circ} 36$, p. 13-40.

Courleux F. (2011). Augmentation de la part des terres agricoles en location : échec ou réussite de la politique foncière. Économie et statistique, $\mathrm{n}^{\circ} 444-445$, p. 39-53.

De Crisenoy C. (1988). De l'origine et du rôle de la politique foncière agricole. Un siècle d'histoire française agricole. Économie rurale, ${ }^{\circ} 184-186$, p. 85-91.

De Crisenoy C. (1979). Modes de faire valoir et tribunaux paritaires (1963-1976). Études sur le fermage. INRA-Esr, $49 \mathrm{p}$.

Darly S., Torre A. (2008). Conflits liés aux espaces agricoles et périmètres de gouvernance en Île-de-France. Géocarrefour, vol. $83, \mathrm{n}^{\circ} 4$, p. 307-319.
Debeaurain J. (2008). Le changement de destination dans le cadre du bail rural. Droit rural, $\mathrm{n}^{\circ} 359$, janvier, étude 6 .

Guéringer A. (2013). Propriété et propriétaires en espaces périurbains ou la gouvernance foncière à l'épreuve des logiques privatives. In Bertrand N. (dir.), Terres agricoles périurbaines: une gouvernance en construction, Paris, Quae, p. 37-54.

Hudault J. (2006). Le Code Napoléon et le droit rural. Droit rural, $\mathrm{n}^{\circ} 342$, avril, étude 17.

Lemonnier É. (2008). Le contrôle du juge sur les actes permettant le changement d'affectation : le contrôle du juge judiciaire. Droit rural, $\mathrm{n}^{\circ} 359$, étude 9 .

Rémy J. (2013). L'exploitation agricole : une institution en mouvement. Demeter, p. 358-384.

Serverin E. (2000). Sociologie du droit. Paris, La Découverte, 118 p. 